\title{
Alcohol use and mental distress as predictors of non-response in a general population health survey: the HUNT study
}

\author{
Fartein Ask Torvik • Kamilla Rognmo • \\ Kristian Tambs
}

Received: 16 August 2010/Accepted: 18 April 2011/Published online: 5 May 2011

(C) The Author(s) 2011. This article is published with open access at Springerlink.com

\begin{abstract}
Purpose To investigate to what degree alcohol use and mental distress are associated with non-response in a population-based health study.

Methods From 1995 to 1997, 91,488 persons were invited to take part in a health study at Nord-Trøndelag, Norway, and the response rate was $69.2 \%$. Demographics were available for everyone. Survey answers from a previous survey were available for most of the participants and a majority of non-participants. In addition, the survey responses from spouses and children of the invitees were used to predict participation in the aforementioned study. Crude and adjusted ORs for a number of predictors, among these alcohol consumption and mental distress, are reported.

Results Both heavy drinkers $(\mathrm{OR}=1.27)$ and abstainers $(\mathrm{OR}=1.64)$ had a higher probability of dropping out in comparison to people who usually do not drink. High levels of mental distress $(\mathrm{OR}=1.84)$ also predicted attrition.

Conclusion Alcohol use and mental distress are moderately associated with non-response, though probably not a major cause, as controlling for other variables weakened the associations. Nevertheless, the moderate but clear underrepresentation at the crude level of people with high alcohol consumption, abstainers and people with poor mental health should be taken into consideration when interpreting results from health surveys.
\end{abstract}

Keywords Non-response · Non-participation · Attrition · Alcohol $\cdot$ Mental distress

F. A. Torvik $(\bowtie) \cdot$ K. Rognmo $\cdot$ K. Tambs Division of Mental Health, Norwegian Institute of Public Health, Nydalen, P.O. Box 4404, 0403 Oslo, Norway

e-mail: fartein.torvik@fhi.no

\section{Introduction}

Using general population health surveys to study alcohol abuse and mental health problems makes it possible to investigate cases that would normally not be included in clinical samples. However, some of the people of interest may not respond. While clinical studies can be criticised for overestimating effect sizes by selecting people with the most severe symptoms, population studies can underestimate effects if important target groups do not respond. Topic-related non-response may threaten external validity by providing non-generalisable prevalence estimates and variable associations [1, 2]. Neither a high response rate [3] nor a demographical weighting of the sample [4] ensure representativeness. To know to what extent results from population studies of alcohol abuse and mental distress are generalisable, it is crucial to investigate whether individuals with such problems are adequately represented.

Alcohol use and non-response

The reported amount of alcohol consumed is consistently found to be considerably lower in population studies than what we know to be true from official sales statistics [5, 6]. While partly a result of underreporting, we do not know to what degree non-response among heavy consumers causes the discrepancy between results from self-report and sale statistics, as seemingly contradictory results have been found on the association between alcohol consumption and participation [2, 4, 7]. There are, however, systematic methodological differences in the studies having investigated non-response.

If one presupposes a "continuum of resistance" to participation, one can assume that reluctant responders, as a point along this continuum, are more similar to 
non-responders than are obliging responders [8] and that characteristics of reluctant responders are accentuated in non-responders. An underrepresentation of people with high alcohol consumption or alcohol-related problems has been found using several methods presupposing a continuum of resistance. When looking at attrition from baseline to follow-up, there was a higher dropout rate among heavy drinkers [9-15]. When comparing early and late responders, late responders were found to consume more alcohol [7, 16, 17], except in one study [5]. Family members of responders and non-responders have been compared and it has been found that less cooperative families were more likely to have alcohol-related problems [18]. Since alcohol consumption is correlated within families, data from family members can act as proxies for an invited person. The studies, which found an underrepresentation of heavy drinkers, either did not find or did not investigate whether abstainers or low consumers were also underrepresented. While research on attrition from baseline to follow-ups provides clear results, it is less evident who participated in the first place, as linear extrapolation to non-responders may not always be correct [5]. In addition, using registries, it has been found that people who are hospitalised or receiving a disability pension due to substance related disorders are less likely to respond to surveys [19, 20]. These people, however, represent extremes and may misuse of other substances than alcohol.

When approaching random samples of persons who did not actually respond to a survey, the opposite results were found. Abstainers, and not heavy drinkers, were significantly overrepresented among non-responders, or nonresponders drank less than responders did [4, 6, 21, 22]. These studies have some methodological limitations. The response rates were low (from 35 to $54 \%$ ), and the data collection changed from questionnaires in the original studies to interviews, thus possibly affecting the validity of self-report data on sensitive topics such as alcohol consumption [23, 24].

\section{Mental distress and non-response}

Quite a few studies find that symptoms of mental health problems are associated with non-response. In clinical trials, the risk of drop-out is higher than average for patients with the most severe symptoms of mental problems [13, 25]. In population health studies, people with mental health problems at baseline are also less likely to respond to follow-up surveys [10, 12, 14, 26, 27]. Drop-out seems to be associated with mortality and a failure to locate the invitees, rather than unwillingness to participate [10, 27]. Distinguishing between early and late responders, one study [16] found that late responders use more psychopharmaceuticals, while another [28] did not find any association between mental health and late response. Using reports from family members as proxies for the invitees, it has been found that less cooperating families score higher on "anxious depression" and neuroticism [18]. Linking registry data from hospital discharges to people invited to participate in a health study, it was found that nonresponders were more likely to have had a psychiatric diagnosis [20, 29].

The opposite result was found among non-participants ( $80 \%$ response rate), who were much less likely to have social phobias [30]. Nevertheless, like other follow-ups of initial non-responders, the data collection method was changed from postal questionnaire to telephone interview. While some type of underrepresentation of mental health problems seems probable, it is unclear how less severe levels of mental distress are related to non-response. It is also unclear as to what degree mental distress uniquely contributes to non-response when controlling for potential confounder variables such as alcohol use, health and demography, all of which are related to mental distress.

Demographical and health variables related to non-response

Other traits commonly found to predict non-participation include smoking [11, 15, 22, 31, 32], high body mass [31, $33]$, unemployment [10, 16, 31], poor subjective health [11, $31]$ and health problems [16, 33-36]. It has repeatedly been found that non-responders are more often male [12, 14, 29, 33 , 35-38], young [10, 11, 26, 29, 36] or old [33, 37, 39], unmarried or divorced [7, 10, 11, 15, 16, 29, 36], live in urban areas [10] and have a low income [7, 10, 15, 36], education $[7,16,29,36,39,40]$ and socioeconomic status $[26,31,35]$. Some studies indicate that people in the upper end of the educational and income range are also somewhat underrepresented [12, 15, 36]. Using the same dataset as the present study, it has been found that non-participation was associated with being male and either young or elderly [37]. In general, results on demographic variables are relatively reliable since such data are often available from public registries including all invited subjects.

\section{Aims of the study}

The aim of the present study is to investigate to what degree general population studies are representative with regard to alcohol use and mental distress. The Nord-Trøndelag Health Study (HUNT) is suitable for investigating the role of these variables as predictors of non-response. It contains large samples with complete demographical registry data, prospective data and kinship data. This permits the examination of alcohol use and mental distress at the same time, while controlling for demographics and health-related variables. 
Supplementary analyses of data from family members can yield some information about those who have never participated. For those who have had a participating spouse, the spouse's data will be used to predict participation. In addition, adolescents were asked questions concerning their parents' behaviour, and their answers will be used to predict their mother's and father's participation. With categorical rather than linear predictor variables, non-participation on both ends of the alcohol use and mental distress scales can be elucidated.

\section{Methods}

Sample and design

From 1984 to 1986 and from 1995 to 1997, all inhabitants in the Norwegian county of Nord-Trøndelag aged 20 years or older were invited to participate in the HUNT study. With each wave, the participants went through a physical examination and completed two questionnaires. The participants then received a letter containing their results, and if necessary, a referral to a doctor.

\section{Main sample}

In the second wave (T2, 1995-1997), 94,188 persons (average age 48.5 years; $50.2 \%$ women) were invited to take part in the health study, with 65,216 persons $(69.2 \%)$ responding to the first questionnaire. The second questionnaire was not used in the present study.

\section{Drop-out sample}

Data from $\mathrm{T} 1$ were used to predict non-participation at $\mathrm{T} 2$ among persons who responded to both questionnaires at $\mathrm{T} 1$ and who were invited at T2. People invited at T2, but not at $\mathrm{T} 1$ were either too young or lived outside the county at that time. People invited at T1, but not at T2 included those who in the meantime between $\mathrm{T} 1$ and $\mathrm{T} 2$ migrated out of the county or passed away. Thus, except for a relatively few persons who died during the weeks from the invitation was prepared to the day of appointment, mortality is not a cause of non-participation. Of the persons invited at $\mathrm{T} 2$, $64,749(68.7 \%)$ had also been invited at T1, out of which $60,079(92.8 \%)$ returned the first questionnaire at $\mathrm{T} 1$ and $50,349(77.8 \%)$ completed the second questionnaire at $\mathrm{T} 1$. Of these, 48,334 (96.0\%) had valid data after imputation (see below) and were included in the prediction of participation at $\mathrm{T} 2$. Of the $\mathrm{T} 2$ non-responders eligible for $\mathrm{T} 1$ participation, $63 \%$ returned the second questionnaire at $\mathrm{T} 1$.
Responses at both $\mathrm{T} 1$ and $\mathrm{T} 2$ from 40,548 persons were used to calculate test-retest stability of the measures.

\section{Spouse sample}

Valid responses from spouses at $\mathrm{T} 2$ were used to predict the invitees' participation at $\mathrm{T} 2$, and cohabitants with children were included in this sample. A total of 53,835 persons ( $57.2 \%$ of the total sample) invited to $\mathrm{T} 2 \mathrm{had}$ a spouse who was also invited to participate in the study. Of the invited spouses, $42,365(78.7 \%)$ participated, of which 40,301 (95.1\%) had valid data on all measures after imputation. Of the non-respondents with an invited spouse, the spouse participated in $43 \%$ of the cases. For the 37,485 couples in which both spouses participated, responses were used to calculate concordance.

\section{Adolescent sample}

At T2, adolescents aged 13 to 19 years old living in the county were also invited to participate in a similar health study called YoungHUNT. Out of 9,917 adolescents invited, 8,984 (90.6\%) responded to the questionnaire. As data were collected during school hours, this sample is thought to be fairly representative of all adolescents within the county. Valid responses were used to predict participation among the adolescents' parents, with only one child per parent being included. If a parent had more than one responding child, the oldest with valid data on exposure to parental alcohol use was chosen. This resulted in a final sample of 6,586 mothers and 6,532 fathers with participating children, of which 6,382 (97\%) mothers and 6,346 (97\%) fathers were included in for the non-response analyses. In the remaining $3 \%$ of the cases, the adolescents had provided incomplete information.

\section{More on HUNT}

Details regarding the methods in the HUNT-1 (T1) [41], HUNT-2 (T2) [37] and YoungHUNT [42] studies have been described elsewhere, and are also described at the HUNT website at http://www.ntnu.no/hunt. The data analysed for this article were slightly different from the data analysed by Holmen and colleagues [37], who removed 1,258 persons who died or moved between the time of the invitation and the health check from the analysis, while the authors of the present article did not have such information. Additionally, 724 persons, primarily above 80 years of age, who were registered as participants in that analysis only provided blood samples and are not considered participants in the present study. 


\section{Measures}

\section{Demographics}

The governmental statistics agency, Statistics Norway, provided demographic data on sex, age, marital status, income, education and urbanicity for all persons invited. For the purposes of this article, age was divided into five categories. Divorce and separation were recoded into the same marital status category, while cohabitants with children were coded as a separate category. Cohabitants without children could not be identified through the public registries. Income was categorised as none, low, medium or high, while education was ordinally scored into five levels. People living in municipalities with township status (all with a population ranging from 10,000 to 20,000 ) were coded as living in towns. There are no larger cities in the county.

\section{Alcohol}

The questionnaires included various alcohol measures. T1 included three questions on alcohol use: drinking frequency, whether one had been drunk during the past 2 weeks, and whether one had been drinking too much in periods of life. These were combined into a summative five point consumption index [total abstainers (9.9\%), no reported drinking over the past two weeks $(42.0 \%)$, some drinking (37.2\%), moderate drinking (8.1\%) and heavy drinking (2.9\%)]. T2 alcohol consumption was measured with a self-report on the number of units drunk during the past two weeks and the number of days drinking in a month. Together with a question on alcohol abstention, five groups were formed [total abstainers (11.2\%), no drinking over the past 2 weeks (24.4\%), some drinking (46.2\%), moderate drinking (men: $12-21$ points on the total of units and drinking days, women: 8-14 points; $13.8 \%$ ) and heavy drinking (4.5\%)]. Adolescents participating in YoungHUNT were asked how often they had seen either of their parents drunk, with answers ranging from "never" to "a few times a week". The wording of this question did not permit distinguishing between fathers and mothers.

\section{Mental distress}

Mental distress was measured at T1 with 12 items related to life satisfaction and mental distress. This measure has been used by Tambs and Moum [43], who regressed these items on the Hopkins Symptom Checklist (SCL-25) [44] in another data material, using regression coefficients to optimise a weighting of the items in a summative indicator. The correlation between the indicator and SCL-25 was 0.82 , and the theta reliability was 0.83 [43]. T2 included two indicators of mental distress (Hospital Anxiety and Depression Scale [45] and CONOR Mental Health Index [46]), which were combined into a single measure $(\alpha=$ 0.89 ). Both the $\mathrm{T} 1$ and $\mathrm{T} 2$ measures were ranked and divided into the following five percentile categories: low $(25 \%)$, average (40\%), elevated (25\%), high (9\%) and very high $(1 \%)$ levels of mental distress.

\section{Health-related measures}

Number of illnesses and disabilities was used as an indicator of physical health. A checklist at $\mathrm{T} 1$ included mobility impairment, impaired vision, impaired hearing, bodily impairments, diabetes, myocardial infarction, angina pectoris, stroke and cerebral haemorrhage. At T2, the checklist was expanded with epilepsy, cancer and "other prolonged illness". The respondents were categorised as having none, one, or two or more illnesses or disabilities, whereas the use of health services was measured at $\mathrm{T} 1$ with questions on whether the respondent had been to a doctor during the past 12 months. Subjective health was measured at $\mathrm{T} 1$ and $\mathrm{T} 2$ with a single item ("How is your health at the moment?"), with four response categories ranging from "poor" to "very good".

\section{Lifestyle-related measures}

Body mass index (BMI) was calculated from height and weight measured at the health check at $\mathrm{T} 1$ and $\mathrm{T} 2$, and was categorised into three groups: normal weight (BMI $<25$, including $1.2 \%$ with BMI $<18.5)$, overweight $(25<\mathrm{BMI}$ $<30)$ and obese $(\mathrm{BMI}>30)$. At T1 and T2, adults were asked whether they were smoking on a daily basis, while adolescents were asked whether their mother and/or their father were smoking at home. Employment status was reported by target persons participating in $\mathrm{T} 1$, with four possible answers: working full time, working part time, working at home and not working. Adolescents were asked whether their parents were separated and, if so, with whom they were living.

\section{Missing data}

In order to avoid excluding persons with a certain proportion of missing data from the analyses, missing data were imputed instrument by instrument, using the maximum likelihood procedure in PASW Statistics 17.0 (formerly known as SPSS) if no more than $75 \%$ of the values were missing for the instrument. For the T1 alcohol measure, $1.1 \%$ of all item scores used were imputed, and $2.6 \%$ of the values used for the $\mathrm{T} 1$ mental distress measure were imputed. A total of $894(1.8 \%)$ persons did not have enough valid items for the alcohol measure to be 
calculated, while $515(1.0 \%)$ were left with missing instrument scores on mental distress. For the spouses at T2, $3.0 \%$ of the values used to calculate the alcohol measure and $4.1 \%$ of the items in the mental distress measure were imputed, with $1,424(3.3 \%)$ and $812(1.9 \%)$ being left with missing instrument scores, respectively. There were no missing data on demographical variables and no imputation was done on adolescent data, as only single item measures were used.

\section{Statistical analyses}

Participation is defined as returning the first questionnaire in HUNT-2. The predictors for non-response to this questionnaire were analysed using binary logistic regression. Four such analyses were performed, one for registry-based demography and one for each of the questionnaires completed by the target person at T1, their spouses at T2 and their adolescent offspring at T2. It is not meaningful to combine data from all sources to predict participation since the group with complete data would only cover a fraction of the variance in willingness to participate. By regressing participation on one variable at a time, crude odds ratios for participation were obtained. Adjusted odds ratios were obtained by entering all predictor variables from a questionnaire together with demography in the same step.

\section{Analysis 1: demography}

First, complete demographic data from public population registries were used to predict non-participation for everyone invited at $\mathrm{T} 2$.

\section{Analysis 2: drop-out from $\mathrm{Tl}$ to $\mathrm{T} 2$}

Second, prospective questionnaire answers from $\mathrm{T} 1$ were used to predict non-response at T2. Non-response was predicted from alcohol use, mental distress, subjective health, physical health, use of health services, body mass index, smoking habits and employment. The degree of stability of participation for a person from $\mathrm{T} 1$ to $\mathrm{T} 2$ was assessed using logistic regression and tetrachoric correlations. The stability of other variables was calculated using Pearson and polychoric correlations.

\section{Analysis 3: spouses}

Third, the spouses' answers at T2 were used to predict participation at $\mathrm{T} 2$ for everyone invited to $\mathrm{T} 2$ who had a participating spouse. For variables that are highly correlated between family members, self-reported family data can be used as proxies to the invited person [18]. Nonresponse was predicted from the spouses' alcohol use, mental distress, smoking habits and physical health. To avoid statistical dependency between the observations, the analyses were run separately for husbands and wives. The degree of dependence of participation between partners was calculated using logistic regression and tetrachoric correlation. Spousal resemblance for other variables was calculated using Pearson and polychoric correlations.

\section{Analysis 4: adolescent children}

Finally, the answers of adolescent offspring reporting directly on their parents' display of alcohol use, smoking or living situation were used to predict non-response among invited parents. The analyses were run separately for mothers and fathers.

\section{Interaction effects}

All potential two-way interaction effects involving alcohol use or mental health were tested. The interaction terms were entered one at a time, together with the other covariates. To limit the family-wise error rate, $\alpha$ was set at 0.01 for the interaction effects.

\section{Software}

PASW Statistics 17.0 by SPSS Inc. was used for all analyses, except for polychoric and tetrachoric correlations, which were calculated using Polycorr 1.1 [47].

\section{Ethics}

The data matching between times of measurement and family members was carried out by Statistics Norway using personal birth identity numbers assigned to every Norwegian citizen. Before the data were returned to the researchers, the identity number was deleted, thus preventing identification of the participants. The Norwegian Data Inspectorate and the Regional Ethics Committee (REC) have approved the HUNT study, and REC approved the present non-response study. Participants gave their written informed consent.

\section{Results}

Analysis 1: demographics

The probability of non-response at $\mathrm{T} 2$ for everyone invited was regressed on sex, age, marital status, income, education and urbanicity in a logistic model, with the results shown in Table 1. High odds ratios (OR) show a high likelihood of non-response. 
Table 1 Demographical predictors of non-response among people invited at $\mathrm{T} 2$

\begin{tabular}{|c|c|c|c|c|}
\hline Variables & $n$ & OR $_{\text {crude }}$ & $\mathrm{OR}_{\mathrm{adj} .}$ & $95 \% \mathrm{CI}_{\mathrm{adj} .}$ \\
\hline \multicolumn{5}{|l|}{ Sex } \\
\hline Female & 47,311 & 1 & 1 & Ref. \\
\hline Male & 46,877 & 1.46 & 1.61 & $1.56-1.67$ \\
\hline$p$ for trend & & $<.001$ & $<.001$ & \\
\hline \multicolumn{5}{|l|}{ Age (years) } \\
\hline $20-29$ & 18,189 & 1 & 1 & Ref. \\
\hline $30-44$ & 25,817 & 0.39 & 0.55 & $0.52-0.58$ \\
\hline $45-59$ & 22,699 & 0.24 & 0.32 & $0.30-0.34$ \\
\hline $60-74$ & 16,255 & 0.19 & 0.15 & $0.14-0.16$ \\
\hline Over 75 & 11,228 & 0.68 & 0.41 & $0.38-0.44$ \\
\hline$p$ for trend & & $<.001$ & $<.001$ & \\
\hline \multicolumn{5}{|l|}{ Marital status } \\
\hline Married & 49,288 & 1 & 1 & Ref. \\
\hline Never married & 23,303 & 3.69 & 2.03 & $1.95-2.13$ \\
\hline Widow & 8,686 & 2.10 & 1.63 & $1.53-1.73$ \\
\hline Divorced or separated & 6,135 & 1.98 & 2.03 & $1.92-2.16$ \\
\hline Cohabitants w/children & 6,776 & 2.01 & 1.41 & $1.32-1.50$ \\
\hline$p$ for trend & & $<.001$ & $<.001$ & \\
\hline \multicolumn{5}{|l|}{ Income } \\
\hline None & 26,129 & 1 & 1 & Ref. \\
\hline Low & 20,425 & 1.05 & 0.64 & $0.60-0.67$ \\
\hline Medium & 34,024 & 0.64 & 0.47 & $0.45-0.50$ \\
\hline High & 13,610 & 0.69 & 0.56 & $0.52-0.60$ \\
\hline$p$ for trend & & $<.001$ & $<.001$ & \\
\hline \multicolumn{5}{|l|}{ Education } \\
\hline Primary & 25,342 & 1 & 1 & Ref. \\
\hline Secondary, lower & 23,749 & 0.71 & 0.69 & $0.66-0.72$ \\
\hline Secondary, higher & 34,178 & 0.99 & 0.59 & $0.56-0.62$ \\
\hline Higher, short & 8,283 & 0.90 & 0.58 & $0.55-0.62$ \\
\hline Higher, long & 2,645 & 1.19 & 0.86 & $0.78-0.94$ \\
\hline$p$ for trend & & $<.001$ & $<.001$ & \\
\hline \multicolumn{5}{|l|}{ Urbanicity } \\
\hline Rural & 34,068 & 1 & 1 & Ref. \\
\hline Town & 60,120 & 1.11 & 1.12 & $1.09-1.16$ \\
\hline$p$ for trend & & $<.001$ & $<.001$ & \\
\hline
\end{tabular}

Crude and adjusted odds ratios

Men were more likely than women to be non-participants and this association was stronger when other demographic variables were controlled. The age of the invitees spanned from 19 to 101 years, and was curvilinearly associated with non-response rates. The probability of nonresponse was highest among people in their 20s and dropped significantly between each age group until the age of 60-74 years. Among the elderly, non-response increased again, and crude and adjusted results revealed the same pattern. When compared to married persons, individuals within all other marital status groups were more likely to be non-responders. Adjusted results showed that those who had never married or were divorced were equally unlikely to respond. Cohabitants with children were more likely to be non-responders than married persons. Income was also related to participation. Adjusted probabilities had a reverse J-shape, with the highest probability of nonresponse in the no income group and the lowest probability of non-response in the medium income group. Education was also non-linearly associated with likelihood of participation. Adjusted risk of non-response decreased from the lowest educational group to the second highest and then increased. Unadjusted results showed that people in the highest educational group were most likely to be nonresponders, while the least educated had the highest nonresponse after adjusting for the other variables. Urbanicity affected participation, with people living in towns being more likely to not respond than those living outside towns.

\section{Analysis 2: drop-out from T1 to T2}

Among those invited to both surveys, subjects responding to both questionnaires at $\mathrm{T} 1$ were less likely to not respond at T2, with an odds ratio of 0.37 (CI 0.36-0.39), which corresponds to a tetrachoric correlation of 0.34 (CI 0.32-0.35). Results from the prediction of non-response at $\mathrm{T} 2$ by questionnaire data from $\mathrm{T} 1$ are shown in Table 2 . Adjusted OR are adjusted for demographics and the remaining $\mathrm{T} 1$ variables.

Although two different measures were used, the stability of alcohol consumption between $\mathrm{T} 1$ and $\mathrm{T} 2$ was strong, with Pearson $r=0.50$ and polychoric $r=0.66$. There was a curvilinear association between alcohol use at $\mathrm{T} 1$ and response at $\mathrm{T} 2$, with abstainers being the most likely to drop out. People with a medium or high consumption also had an elevated risk of dropping out, while people with a low consumption were significantly less likely to drop out than do people with no consumption. The unadjusted nonresponse rate at $\mathrm{T} 2$ was $16.1 \%$ among persons consuming small amounts of alcohol at T1, compared to $27.9 \%$ among abstainers and $22.9 \%$ among heavy drinkers. When these results were adjusted for demographics and the other variables in this analysis, the same pattern remained, albeit at a somewhat reduced magnitude. Nonetheless, both extremes still predicted non-response compared to low consumption.

The categorised measures of mental distress showed a lower correlation between $\mathrm{T} 1$ and $\mathrm{T} 2$ than did alcohol consumption, with Pearson $r=0.44$ and polychoric $r=0.49$. Unadjusted, mental distress at $\mathrm{T} 1$ was curvilinearly associated with non-response at $\mathrm{T} 2$, and people with very high levels of mental distress were most likely to drop out. People with moderate levels of mental distress were a little less likely to drop out than do people with the lowest 
Table 2 Answers at $\mathrm{T} 1$ as predictors for non-response at T2, among $\mathrm{T} 1$ responders re-invited at $\mathrm{T} 2$

\begin{tabular}{|c|c|c|c|c|}
\hline Variables & $n$ & $\mathrm{OR}_{\text {crude }}$ & $\mathrm{OR}_{\mathrm{adj}}$ & $95 \% \mathrm{CI}_{\mathrm{adj}}$. \\
\hline \multicolumn{5}{|c|}{ Alcohol consumption, T1 } \\
\hline Abstainer & 4,782 & 1.64 & 1.41 & $1.30-1.52$ \\
\hline No & 20,285 & 1 & 1 & Ref. \\
\hline Some & 17,959 & 0.83 & 0.96 & $0.90-1.02$ \\
\hline Medium & 3,900 & 1.16 & 1.10 & $1.00-1.21$ \\
\hline High & 1,408 & 1.27 & 1.13 & $0.98-1.30$ \\
\hline$p$ for trend & & $<.001$ & $<.001$ & \\
\hline \multicolumn{5}{|c|}{ Mental distress, T1 } \\
\hline Low & 12,126 & 1 & 1 & Ref. \\
\hline Average & 19,417 & 0.92 & 0.94 & $0.88-1.00$ \\
\hline Elevated & 12,034 & 0.97 & 0.94 & $0.88-1.01$ \\
\hline High & 4,285 & 1.24 & 1.04 & $0.94-1.15$ \\
\hline Very high & 472 & 1.84 & 1.21 & $0.96-1.51$ \\
\hline$p$ for trend & & $<.001$ & .018 & \\
\hline \multicolumn{5}{|c|}{ Subjective health, T1 } \\
\hline Very good & 8,040 & 1 & 1 & Ref. \\
\hline Good & 29,712 & 0.99 & 0.90 & $0.84-0.97$ \\
\hline Not so good & 10,009 & 1.41 & 0.93 & $0.84-1.02$ \\
\hline $\mathrm{Bad}$ & 573 & 2.31 & 1.17 & $0.95-1.45$ \\
\hline$p$ for trend & & $<.001$ & .002 & \\
\hline \multicolumn{5}{|l|}{ Smoking, T1 } \\
\hline No & 31,710 & 1 & 1 & Ref. \\
\hline Yes & 16,624 & 1.29 & 1.59 & $1.51-1.68$ \\
\hline$p$ for trend & & $<.001$ & $<.001$ & \\
\hline \multicolumn{5}{|c|}{ Illnesses and disabilities, T1 } \\
\hline None & 34,084 & 1 & 1 & Ref. \\
\hline One & 10,795 & 1.37 & 0.99 & $0.92-1.05$ \\
\hline More than one & 3,455 & 2.29 & 1.15 & $1.04-1.27$ \\
\hline$p$ for trend & & $<.001$ & .004 & \\
\hline \multicolumn{5}{|l|}{ Body mass, T1 } \\
\hline Normal & 26,479 & 1 & 1 & Ref. \\
\hline Overweight & 17,067 & 1.20 & 1.10 & $1.04-1.16$ \\
\hline Obese & 4,788 & 1.77 & 1.47 & $1.36-1.60$ \\
\hline$p$ for trend & & $<.001$ & $<.001$ & \\
\hline \multicolumn{5}{|c|}{ Use of health services, T1 } \\
\hline Yes & 36,630 & 1 & 1 & Ref. \\
\hline No & 11,704 & 1.17 & 1.17 & $1.11-1.24$ \\
\hline$p$ for trend & & $<.001$ & $<.001$ & \\
\hline \multicolumn{5}{|l|}{ Employment, T1 } \\
\hline Full time & 22,964 & 1 & 1 & Ref. \\
\hline Part time & 9,549 & 0.74 & 0.92 & $0.84-1.00$ \\
\hline At home & 6,428 & 1.22 & 1.24 & $1.13-1.36$ \\
\hline No & 9,393 & 2.82 & 1.56 & $1.44-1.69$ \\
\hline$p$ for trend & & $<.001$ & $<.001$ & \\
\hline
\end{tabular}

Adjusted results are adjusted for demographics and the remaining T1 variables scores. When controlling for demographics and the other variables in this analysis, the strength of the associations was strongly reduced.

Subjective health was fairly stable between $\mathrm{T} 1$ and $\mathrm{T} 2$, with correlations of Pearson $r=0.46$ and polychoric $r=0.57$. Persons who rated their health as "bad" were most likely to drop out. Adjusted results also pointed to an increased probability of drop-out among those with "very good" subjective health compared to those with "good". The stability of smoking between $\mathrm{T} 1$ and T2 for responders was Pearson $r=0.70$ and tetrachoric $r=$ 0.91. Corresponding correlation for body mass index was Pearson $r=0.83$, for physical illness Pearson $r=0.28$ and polychoric $r=0.43$ and for employment polychoric $r=0.55$. Smokers, overweight and obese persons, persons with poor physical health, persons who did not use health services, and unemployed persons were less likely to participate.

The only statistically significant interaction effect at $\alpha=0.01$ level was between alcohol use and employment status (Wald $=28.34, p=.005$ ). Logistic regression stratified by employment showed that the probability of drop-out among abstainers varied between employment groups, and was higher in the group who worked part time $(\mathrm{OR}=1.63$, CI $1.30-2.03)$, at home $(\mathrm{OR}=1.37, \mathrm{CI}$ $1.14-1.64)$, and among the unemployed (OR $=1.39$, CI 1.23-1.56) than among those with a full-time job $(\mathrm{OR}=1.15$, CI $0.95-1.38)$ when compared to people with no consumption within each employment group. No statistically significant interactions were found between alcohol use or mental distress and any of the other variables. The $p$ value for the interaction between alcohol use and mental distress was 0.201 .

Analysis 3: spouse data

The odds ratio for not responding at $\mathrm{T} 2$ if the spouse responded at T2 was 0.09 (95\% CI 0.08-0.09), corresponding to a tetrachoric correlation of 0.70 . The odds ratios for non-participation among persons with valid spousal data from T2 are shown in Table 3.

The interspousal correlation for alcohol consumption was Pearson $r=0.58$ and polychoric $r=64$. The crude association between response and the spouse's alcohol consumption fell short of significance for both men and women. When mental distress, smoking, illness and demographics were added to the analyses, this association became significant for men, i.e. the male response could be predicted from their wives' alcohol consumption level. Men whose wives consumed some alcohol were less likely to be non-responders. 
Table 3 Spouse answers at T2 as predictors for non-response at T2, among invitees with participating spouses

\begin{tabular}{|c|c|c|c|c|c|c|c|c|}
\hline \multirow[t]{2}{*}{ Variables } & \multicolumn{4}{|c|}{ Women (invitee, male spouse) } & \multicolumn{4}{|c|}{ Men (invitee, female spouse) } \\
\hline & $n$ & $\mathrm{OR}_{\text {crude }}$ & $\mathrm{OR}_{\text {adj. }}$ & $95 \% \mathrm{CI}_{\text {adj. }}$ & $n$ & $\mathrm{OR}_{\text {crude }}$ & $\mathrm{OR}_{\text {adj. }}$ & $95 \% \mathrm{CI}_{\mathrm{adj}}$ \\
\hline \multicolumn{9}{|l|}{ Alcohol, spouse } \\
\hline Abstainer & 1,518 & 0.99 & 1.00 & $0.80-1.24$ & 2,997 & 0.86 & 1.00 & $0.87-1.14$ \\
\hline No & 3,698 & 1 & 1 & Ref. & 6,109 & 1 & 1 & Ref. \\
\hline Some & 10,369 & 0.85 & 0.87 & $0.75-1.01$ & 8,239 & 0.95 & 0.86 & $0.78-0.94$ \\
\hline Medium & 2,840 & 1.02 & 1.03 & $0.85-1.24$ & 2,709 & 0.97 & 0.89 & $0.78-1.02$ \\
\hline High & 946 & 1.00 & 1.02 & $0.78-1.34$ & 849 & 0.91 & 0.91 & $0.74-1.13$ \\
\hline$p$ for trend & & .058 & .108 & & & .184 & .021 & \\
\hline \multicolumn{9}{|c|}{ Mental distress, spouse } \\
\hline Low & 5,100 & 1 & 1 & Ref. & 5,282 & 1 & 1 & Ref. \\
\hline Average & 8,165 & 1.10 & 1.11 & $0.97-1.27$ & 8,239 & 0.90 & 0.92 & $0.83-1.01$ \\
\hline Elevated & 4,491 & 1.15 & 1.18 & $1.01-1.38$ & 5,032 & 0.96 & 1.01 & $0.91-1.13$ \\
\hline High & 1,495 & 1.56 & 1.57 & $1.29-1.93$ & 2,104 & 1.01 & 1.06 & $0.92-1.22$ \\
\hline Very high & 147 & 1.42 & 1.42 & $0.80-2.51$ & 246 & 1.14 & 1.22 & $0.86-1.74$ \\
\hline$p$ for trend & & $<.001$ & $<.001$ & & & .149 & .081 & \\
\hline \multicolumn{9}{|l|}{ Smoking, spouse } \\
\hline No & 14,370 & 1 & 1 & Ref. & 14,940 & 1 & 1 & Ref. \\
\hline Yes & 5,028 & 1.30 & 1.30 & $1.15-1.46$ & 5,963 & 1.37 & 1.31 & $1.20-1.43$ \\
\hline$p$ for trend & & $<.001$ & $<.001$ & & & $<.001$ & $<.001$ & \\
\hline \multicolumn{9}{|c|}{ Illnesses and disabilities, spouse } \\
\hline None & 14,240 & 1 & 1 & Ref. & 16,622 & 1 & 1 & Ref. \\
\hline One & 3,153 & 0.81 & 0.79 & $0.67-0.93$ & 3,020 & 0.79 & 0.87 & $0.77-0.98$ \\
\hline Two or more & 2,005 & 0.91 & 0.84 & $0.69-1.03$ & 1,261 & 0.72 & 0.81 & $0.67-0.98$ \\
\hline$p$ for trend & & .025 & .010 & & & $<.001$ & .015 & \\
\hline
\end{tabular}

Crude results and results adjusted for demographics and the other variables in the table

Mental distress correlated Pearson $r=0.23$ and polychoric $r=0.27$ between spouses. Poor mental health in the spouse predicted a lower probability of response for women, i.e. if a man reported poor mental health it was then more likely that his wife did not participate. The crude and adjusted results were approximately the same, and a similar tendency was found among men, although it was not significant.

Smoking correlated Pearson $r=0.32$ or tetrachoric $r=0.51$ between spouses. Having a smoking spouse was associated with an increased probability of non-response, both for men and for women, with approximately the same estimates at a crude level when all the other variables were controlled. The correlation between spouses for physical illness or disability was Pearson $r=0.16$ and tetrachoric $r=0.28$. Men and women who had an ill or disabled spouse were less likely to be non-responders to the health survey than those who did not.

None of the potential interaction effects between alcohol use or mental distress and any of the other variables in the analysis or the invitee's demography was significant. The $p$ value for the interaction between alcohol use and mental distress was 0.437 for female target persons and 0.921 for male target persons.

Analysis 4: adolescent children

The results of a logistic regression of data from children reporting on parental display of alcohol use, housing situation and smoking are presented in Table 4, crude and controlled for demographics.

Crude results showed that child report of having seen ones parents drunk was a significant predictor of both maternal and paternal response. A cross-tabulation revealed that nonresponse rates were lowest among those parents whose children reported that they had never seen them drunk. Within this group, $14.1 \%$ of invited mothers and $21.7 \%$ of invited fathers did not respond. Mothers and fathers of children who reported that they had seen their parents drunk several times a week had non-response rates of 22.2 and $34.5 \%$, respectively. All groups had a significantly higher probability of non-response than the reference groups who had never seen their parents drunk. The odds ratio of non-response for each group was rising with an increasing frequency of seeing their parents 
Table 4 Adolescent children's answers at T2 as predictors for participation at T2 among parents with participating children

\begin{tabular}{|c|c|c|c|c|c|c|c|c|}
\hline \multirow[t]{2}{*}{ Variables } & \multicolumn{4}{|c|}{ Mothers } & \multicolumn{4}{|c|}{ Fathers } \\
\hline & $n$ & OR $_{\text {crude }}$ & $\mathrm{OR}_{\mathrm{adj}}$ & $95 \% \mathrm{CI}_{\text {adj. }}$ & $n$ & OR $_{\text {crude }}$ & $\mathrm{OR}_{\text {adj. }}$ & $95 \% \mathrm{CI}_{\text {adj. }}$ \\
\hline \multicolumn{9}{|l|}{ Seen parents drunk } \\
\hline Never & 2,340 & 1 & 1 & Ref. & 2,162 & 1 & 1 & Ref. \\
\hline A few times & 2,567 & 1.25 & 1.12 & $0.95-1.32$ & 2,376 & 1.17 & 1.06 & $0.91-1.22$ \\
\hline A few times a year & 1,465 & 1.36 & 1.18 & $0.98-1.42$ & 1,378 & 1.32 & 1.18 & $1.00-1.39$ \\
\hline A few times a month & 373 & 1.69 & 1.28 & $0.96-1.71$ & 345 & 1.73 & 1.30 & $1.00-1.68$ \\
\hline A few times a week & 87 & 1.73 & 1.23 & $0.72-2.11$ & 85 & 1.89 & 1.22 & $0.75-1.97$ \\
\hline$p$ for trend & & $<.001$ & .309 & & & $<.001$ & .159 & \\
\hline \multicolumn{9}{|l|}{ Housing } \\
\hline Parents living together & 5,467 & 1 & 1 & Ref. & 5,371 & 1 & 1 & Ref. \\
\hline Living with mother & 994 & 2.20 & 1.38 & $1.07-1.79$ & 552 & 2.50 & 1.73 & $1.33-2.26$ \\
\hline Living with father & 147 & 2.71 & 1.83 & $1.19-2.79$ & 237 & 1.70 & 1.14 & $0.80-1.63$ \\
\hline Other & 224 & 1.70 & 1.19 & $0.82-1.73$ & 186 & 2.41 & 1.66 & $1.16-2.38$ \\
\hline$p$ for trend & & $<.001$ & .018 & & & $<.001$ & $<.001$ & \\
\hline \multicolumn{9}{|l|}{ Smoking } \\
\hline No & 4,161 & 1 & 1 & Ref. & 4,409 & 1 & 1 & Ref. \\
\hline Yes & 2,671 & 1.85 & 1.48 & $1.29-1.70$ & 1,937 & 1.70 & 1.61 & $1.42-1.83$ \\
\hline$p$ for trend & & $<.001$ & $<.001$ & & & $<.001$ & $<.001$ & \\
\hline
\end{tabular}

Adjusted results are adjusted for demographics and the other variables in the table

drunk. When also controlling for living situation, parental smoking and demographics, reports of having seen one's parents drunk were not a significant predictor of their nonparticipation, though there were tendencies in that direction.

Housing was related to non-response. Parents who did not live together were significantly less likely to participate than parents living together, with non-custodial parents seeming to have the lowest response rates. When controlling for other variables, the association became weaker, although all groups still had a higher probability of nonresponse than couples, with the exception of single parent fathers. Mothers and fathers who were smokers, as reported by their adolescent children, were significantly less likely to participate, both judging from crude and adjusted results. Child report of parental smoking was a reliable measure since they corresponded with self-report among parents who participated in $93 \%$ of the cases. Full consistency should not be expected because the wording was different.

No statistically significant interaction effects were found between parental displays of alcohol use and any of the other variables in the analysis.

\section{Discussion}

\section{Alcohol use}

Heavy drinkers were underrepresented compared to people with low consumption, which replicated previous studies
[9-15]. An association between parental response and display of alcohol use reported independently by adolescent children supports this finding. In addition, people who defined themselves as abstainers were considerably more likely to drop out than low consumers. Thus, this study confirms the suspicion that abstainers are also underrepresented [2, 4, 7, 21, 22]. We were able to find nonresponse on both ends of the scale because alcohol use was analysed with several categories instead of linearly or dichotomously. As in previous research, the effect of alcohol use on participation appears to be modest. Using spousal consumption as an approximation to the invitee's consumption yielded unclear results, but also indicated curvilinearity. As the associations were weakened when controlling for other variables, alcohol use is probably not a major cause of non-response.

It has been suggested that heavy drinkers may be underrepresented because they are difficult to reach (wrong address, not at home, etc.) [2, 4].That some people avoid alcohol specifically as a topic [4] could not be the reason here, as HUNT was not presented as an alcohol study. Both abstention and heavy drinking are deviations from social norms and could be associated with personal characteristics that influence the willingness and opportunity to participate. For instance, people labelling themselves as being total abstainers in comparison to people who usually do not drink have higher symptom scores for anxiety and depression [48], smaller social networks [49] and are more religious [50]. Among abstainers, there could also be some 
sick quitters, who are more similar to heavy drinkers. A lack of interest in obtaining a health check or in research could be a reason for non-response, and neither heavy drinking nor abstention indicates a special interest in health. People who feel like outsiders may also be less motivated to contribute to research as predicted by social exchange theory [51].

\section{Mental distress}

High levels of mental distress predicted non-response, and this association was greatly reduced when controlling for other variables. Both findings are in line with previous research [14, 16, 26, 27, 29]. A large sample such as this was needed in order to reach statistical significance. The inclusion of a "very high" mental distress group might have made the results clearer. There was an unforeseen tendency for people with below-average mental distress not to respond, compared to people with average mental distress. Mental health problems in participating husbands also predicted non-response among women.

Increased rates of non-response among mentally distressed people may reflect difficulties in locating them, that they do not have a surplus of energy to participate, social anxiety, or that they are not interested in their health or in contributing to research for whatever reason. One may speculate that the slight tendency for clearly mentally healthy people not to respond is caused by a lack of worrying, and therefore a lack of interest in their own health. As mental distress was only weakly correlated between partners, spouses cannot be seen as an approximation to the invitee in this regard. Since women's participation can nevertheless be predicted from their husband's level of mental distress, it is likely that some other factors related to the husband, family or resource situation affects the wives of the mentally distressed, for example the burden of care.

\section{Demography and health}

Results on demographics and health-related variables are generally in agreement with previous research. It is interesting to note that the highest participation was in the groups, which scored the second highest on the indicators of socioeconomic status (income and education). Smoking was a stable predictor for non-participation across all analyses. The other health-related variables, physical health, subjective health, use of health services and body mass were all independently associated with response.

Slightly different results from previous research may be due to various methodologies such as the categorisation of all variables and sample size or because surveys actually have different patterns of non-response. As the HUNT study was introduced to the participants as a health study, which offered a health check, one would expect participation to be associated with an interest in health, as our results show.

The moderate correlation between participation in $\mathrm{T} 1$ and $\mathrm{T} 2$ indicates that there is a good chance of re-recruiting participants lost between waves.

Strengths and weaknesses

In both the drop-out and partner analyses, persons who never participated and couples in which none of the spouses participated could not be analysed. Double nonresponse may indicate a narrowing of variance in willingness to participate, thus implying that estimates from these two analyses may be somewhat downwardly biased. This could be particularly noticeable in the spouse analysis, in which spouse participation is highly interdependent. That analysis also provided the least clear-cut results. Drinking and mental distress appear to be somewhat more widespread among drop-outs and probably even more so among persons who never participated. In another Norwegian study [19] only $18 \%$ of people who received a pension for substance use related diagnoses participated. It is, however, unclear to what extent double non-response attenuated the effect sizes. Those receiving a disability pension only represent the most extreme cases, and also, many of those people may be abusing other substances than alcohol.

The analysis of demographic data from registries as well as data from the adolescent study stays clear of the problem with double non-responders. Only a fraction of the invitees were represented as parents in the adolescent sample, but for those with children aged 13-19 this sample is quite complete, with a $91 \%$ response rate. It was not possible, however, to distinguish whether the adolescents had seen their mother or father drunk, which may be a limitation to the study and probably attenuate the predictive power of this variable for the individual participation of each of the parents.

The high partner correlation for participation in this study may partly reflect that persons from the same households were invited to come to the site of the health examination at the same time. Because of this, using information about partners as proxies to information about the target person turned out to be problematic. Perhaps a lower partner correlation should be expected for purely postal questionnaire studies.

As the data are self-reported, misclassification could occur, thereby leading to an underestimation of the associations with non-response. For alcohol use, underreporting occurs [52], but as the alcohol measures are highly correlated between $\mathrm{T} 1$ and $\mathrm{T} 2$ and between spouses, misclassification is either modest or consistent. Misclassification 
could underestimate effect sizes more among heavy drinkers than among abstainers, as abstention is a more easily defined and measured endpoint.

The results may be more generalisable to similar health studies than to postal surveys, as the participants had to travel to an examination site and spend more time than if they were only to answer a questionnaire, and they may have been motivated by receiving the health check. Even so, as the results for most variables replicated research on non-response in different types of studies, it may also be that the same variables predict participation across methods.

\section{Implications and conclusion}

Although each single odds ratio for non-participation primarily takes moderate values, combinations of elevated risk, calculated as products of two or more odds ratios, may yield a very high risk of non-response. For example, mentally distressed, young, single males will be severely underrepresented in a study such as HUNT and should be studied otherwise.

It seems like people who report alcohol and mental distress problems are less likely to respond to a health survey such as HUNT, though only moderately, and this association is rather weak when controlling for other variables. It is worth noting that abstainers are the alcohol consumption group with the highest attrition, and that there is a considerable difference between this group and the no consumption group. The large sample size provided good estimates of associations between response and other variables. The demographic data are particularly reliable.

The underrepresentation of specific groups is important to consider when interpreting future and previous research in HUNT, other general population-based health studies, and questionnaire studies in general. Results on alcohol consumption and mental health may be affected by nonresponse. Nevertheless, the selective non-response rates observed in this study are of a modest magnitude, which makes us believe that these kinds of studies can be suitable for investigating risk and protective factors in relation to causes or consequences of alcohol use and mental distress, or at the very least, that the HUNT study is suitable for such purposes. Still, estimates of prevalence or incidence may be somewhat biased.

Acknowledgments Nord-Trøndelag Health Study (The HUNT Study) is a collaboration between HUNT Research Centre (Faculty of Medicine, Norwegian University of Science and Technology NTNU), Nord-Trøndelag County Council and The Norwegian Institute of Public Health. This work was supported by grants from The Research Council of Norway (project number 190519), and forms part of a doctoral thesis to be submitted to the Department of Psychology, University of Oslo.
Open Access This article is distributed under the terms of the Creative Commons Attribution Noncommercial License which permits any noncommercial use, distribution, and reproduction in any medium, provided the original author(s) and source are credited.

\section{References}

1. Miller RB, Wright DW (1995) Detecting and correcting attrition bias in longitudinal family research. J Marriage Fam 57:921-929

2. Caetano R (2001) Non-response in alcohol and drug surveys: a research topic in need of further attention. Addiction 96: $1541-1545$

3. der Wiel AB, van EE, de Craen AJ, Gussekloo J, Lagaay AM, Knook DL, Westendorp RG (2002) A high response is not essential to prevent selection bias: results from the Leiden 85-plus study. J Clin Epidemiol 55:1119-1125

4. Lahaut VM, Jansen HA, van de Mheen D, Garretsen HF (2002) Non-response bias in a sample survey on alcohol consumption. Alcohol Alcohol 37:256-260

5. Lahaut VM, Jansen HA, van de Mheen D, Garretsen HF, Verdurmen JE, van DA (2003) Estimating non-response bias in a survey on alcohol consumption: comparison of response waves. Alcohol Alcohol 38:128-134

6. Lemmens PH, Tan ES, Knibbe RA (1988) Bias due to nonresponse in a Dutch survey on alcohol consumption. Br J Addict 83:1069-1077

7. Zhao J, Stockwell T, Macdonald S (2009) Non-response bias in alcohol and drug population surveys. Drug Alcohol Rev 28: 648-657

8. Lin IF, Schaeffer NC (1995) Using survey participants to estimate the impact of nonparticipation. Public Opin Quart 59: 236-258

9. Buckner JD, Schmidt NB, Lang AR, Small JW, Schlauch RC, Lewinsohn PM (2008) Specificity of social anxiety disorder as a risk factor for alcohol and cannabis dependence. J Psychiatr Res 42:230-239

10. de Graaf R, Bijl RV, Smit F, Ravelli A, Vollebergh WA (2000) Psychiatric and sociodemographic predictors of attrition in a longitudinal study: The Netherlands Mental Health Survey and Incidence Study (NEMESIS). Am J Epidemiol 152:1039-1047

11. Goldberg M, Chastang JF, Zins M, Niedhammer I, Leclerc A (2006) Health problems were the strongest predictors of attrition during follow-up of the GAZEL cohort. J Clin Epidemiol 59: 1213-1221

12. Heath AC, Howells W, Kirk KM, Madden PA, Bucholz KK, Nelson EC, Slutske WS, Statham DJ, Martin NG (2001) Predictors of non-response to a questionnaire survey of a volunteer twin panel: findings from the Australian 1989 twin cohort. Twin Res 4:73-80

13. Nomamiukor N, Brown ES (2009) Attrition factors in clinical trials of comorbid bipolar and substance-related disorders. J Affect Disord 112:284-288

14. Tambs K, Ronning T, Prescott CA, Kendler KS, ReichbornKjennerud T, Torgersen S, Harris JR (2009) The Norwegian Institute of Public Health twin study of mental health: examining recruitment and attrition bias. Twin Res Hum Genet 12:158-168

15. Thygesen LC, Johansen C, Keiding N, Giovannucci E, Gronbaek M (2008) Effects of sample attrition in a longitudinal study of the association between alcohol intake and all-cause mortality. Addiction 103:1149-1159

16. Korkeila K, Suominen S, Ahvenainen J, Ojanlatva A, Rautava P, Helenius H, Koskenvuo M (2001) Non-response and related 
factors in a nation-wide health survey. Eur $\mathrm{J}$ Epidemiol 17: 991-999

17. Kypri K, Stephenson S, Langley J (2004) Assessment of nonresponse bias in an internet survey of alcohol use. Alcohol Clin Exp Res 28:630-634

18. Vink JM, Willemsen G, Stubbe JH, Middeldorp CM, Ligthart RS, Baas KD, Dirkzwager HJ, de Geus EJ, Boomsma DI (2004) Estimating non-response bias in family studies: application to mental health and lifestyle. Eur J Epidemiol 19:623-630

19. Knudsen AK, Hotopf M, Skogen JC, Overland S, Mykletun A (2010) The health status of nonparticipants in a population-based health study: the Hordaland health study. Am J Epidemiol 172: 1306-1314

20. Bergman P, Ahlberg G, Forsell Y, Lundberg I (2010) Non-participation in the second wave of the PART study on mental disorder and its effects on risk estimates. Int J Soc Psychiatry 56:119-132

21. Cranford JA, McCabe SE, Boyd CJ, Slayden J, Reed MB, Ketchie JM, Lange JE, Scott MS (2008) Reasons for nonresponse in a web-based survey of alcohol involvement among first-year college students. Addict Behav 33:206-210

22. Hill A, Roberts J, Ewings P, Gunnell D (1997) Non-response bias in a lifestyle survey. J Public Health Med 19:203-207

23. Gmel G (2000) The effect of mode of data collection and of nonresponse on reported alcohol consumption: a split-sample study in Switzerland. Addiction 95:123-134

24. Greenfield TK, Midanik LT, Rogers JD (2000) Effects of telephone versus face-to-face interview modes on reports of alcohol consumption. Addiction 95:277-284

25. Haapea M, Miettunen J, Veijola J, Lauronen E, Tanskanen P, Isohanni M (2007) Non-participation may bias the results of a psychiatric survey: an analysis from the survey including magnetic resonance imaging within the Northern Finland 1966 Birth Cohort. Soc Psychiatry Psychiatr Epidemiol 42:403-409

26. Batty GD, Gale CR (2009) Impact of resurvey non-response on the associations between baseline risk factors and cardiovascular disease mortality: prospective cohort study. J Epidemiol Community Health 63:952-955

27. Eaton WW, Anthony JC, Tepper S, Dryman A (1992) Psychopathology and attrition in the epidemiologic catchment area surveys. Am J Epidemiol 135:1051-1059

28. Etter JF, Perneger TV (1997) Analysis of non-response bias in a mailed health survey. J Clin Epidemiol 50:1123-1128

29. Lundberg I, Damstrom TK, Hallstrom T, Forsell Y (2005) Determinants of non-participation, and the effects of non-participation on potential cause-effect relationships, in the PART study on mental disorders. Soc Psychiatry Psychiatr Epidemiol 40: $475-483$

30. Furmark T, Tillfors M, Everz P, Marteinsdottir I, Gefvert O, Fredrikson M (1999) Social phobia in the general population: prevalence and sociodemographic profile. Soc Psychiatry Psychiatr Epidemiol 34:416-424

31. van Loon AJ, Tijhuis M, Picavet HS, Surtees PG, Ormel J (2003) Survey non-response in the Netherlands: effects on prevalence estimates and associations. Ann Epidemiol 13:105-110

32. Wall M, Teeland L (2004) Non-participants in a preventive health examination for cardiovascular disease: characteristics, reasons for non-participation, and willingness to participate in the future. Scand J Prim Health Care 22:248-251

33. Bjerkeset O, Romundstad P, Evans J, Gunnell D (2008) Association of adult body mass index and height with anxiety, depression, and suicide in the general population: the HUNT study. Am J Epidemiol 167:193-202

34. Chatfield MD, Brayne CE, Matthews FE (2005) A systematic literature review of attrition between waves in longitudinal studies in the elderly shows a consistent pattern of dropout between differing studies. J Clin Epidemiol 58:13-19

35. Martikainen P, Laaksonen M, Piha K, Lallukka T (2007) Does survey non-response bias the association between occupational social class and health? Scand J Public Health 35:212-215

36. Sogaard AJ, Selmer R, Bjertness E, Thelle D (2004) The Oslo Health Study: the impact of self-selection in a large, populationbased survey. Int J Equity Health 3:3

37. Holmen J, Midthjell K, Kruger O, Langhammer A, Holmen TL, Bratberg GH, Vatten L, Lund-Larsen PG (2003) The NordTrøndelag Health Study 1995-97 (HUNT 2): Objectives, contents, methods and participation. Nor Epidemiol 13:19-32

38. Hara M, Sasaki S, Sobue T, Yamamoto S, Tsugane S (2002) Comparison of cause-specific mortality between respondents and nonrespondents in a population-based prospective study: ten-year follow-up of JPHC Study Cohort I. Japan Public Health Center. J Clin Epidemiol 55:150-156

39. Stranges S, Notaro J, Freudenheim JL, Calogero RM, Muti P, Farinaro E, Russell M, Nochajski TH, Trevisan M (2006) Alcohol drinking pattern and subjective health in a population-based study. Addiction 101:1265-1276

40. Pettersson C, Linden-Bostrom M, Eriksson C (2009) Reasons for non-participation in a parental program concerning underage drinking: a mixed-method study. BMC Public Health 9:478

41. Holmen J, Midthjell K, Bjartveit K, Gjort.P.F, Lund-Larsen PG, Moum T, Næss S, Waaler HT (1990) The Nord-Trøndelag Health Survey 1984-86. Purpose, background and methods. Participation, non-participation and frequency distributions. Helsetjenesteforskning, Verdal

42. Holmen TL (2000) Smoking and health in adolescence. dissertation, Norwegian University of Science and Technology, Trondheim

43. Tambs K, Moum T (1993) Low genetic effect and age-specific family effect for symptoms of anxiety and depression in nuclear families, halfsibs and twins. J Affect Disord 27:183-195

44. Derogatis LR, Lipman RS, Rickels K, Uhlenhuth EH, Covi L (1974) The Hopkins Symptom Checklist (HSCL): a self-report symptom inventory. Behav Sci 19:1-15

45. Zigmond AS, Snaith RP (1983) The hospital anxiety and depression scale. Acta Psychiatr Scand 67:361-370

46. Søgaard AJ, Bjelland I, Tell GS, Røysamb E (2003) A comparison of the CONOR Mental Health Index to the HSCL-10 and HADS. Measuring mental health status in The Oslo Health Study and the Nord-Trøndelag Health Study. Nor Epidemiol 13: 279-284

47. Uebersax JS (2007) User Guide for POLYCORR 1.1. Statistical methods for rater agreement web site. http://john-uebersax.com/ stat/xpc.htm. Accessed 05 April 2011

48. Skogen JC, Harvey SB, Henderson M, Stordal E, Mykletun A (2009) Anxiety and depression among abstainers and low-level alcohol consumers. The Nord-Trøndelag Health Study. Addiction 104:1519-1529

49. Graham K (1998) Alcohol abstention among older adults: Reasons for abstaining and characteristics of abstainers. Addiction Res 6:473-487

50. Michalak L, Trocki K, Bond J (2007) Religion and alcohol in the US National Alcohol Survey: how important is religion for abstention and drinking? Drug Alcohol Depend 87:268-280

51. Dillman DA (2000) Mail and Internet surveys: the Tailored Design Method. Wiley, New York

52. Lahaut VMHCJ, Jansen HAM, van de Mheen D, Garretsen HFL (2003) Comparison of two formats of the weekly recall and quantity frequency measures. Journal Subst Use 8:164-169 